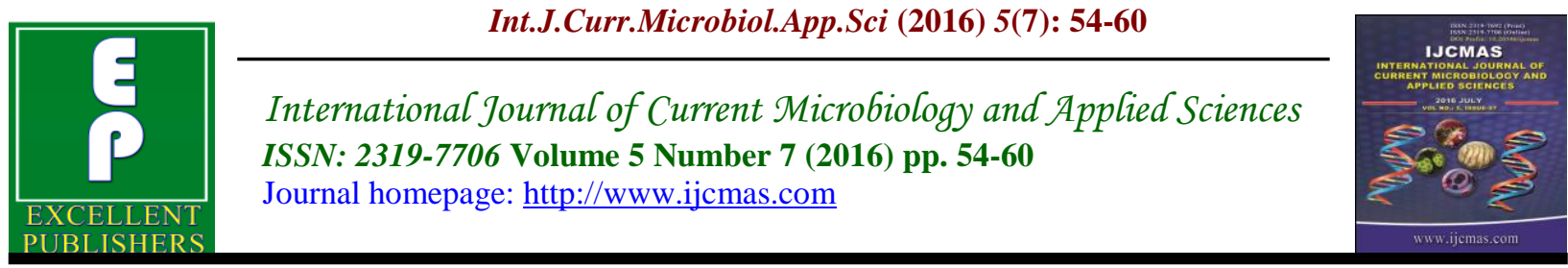

Original Research Article

http://dx.doi.org/10.20546/ijcmas.2016.507.004

\title{
Speciation of Candida Isolated from Various Clinical Samples and their Antifungal Susceptibility Profile in a Tertiary Care Hospital
}

\author{
M.V. Natasha ${ }^{1 *}$, Y. Raksha ${ }^{2}$ and B.V. Navaneeth ${ }^{3}$ \\ ${ }^{1}$ Department of Microbiology, Gulbarga Institute Of Medical Sciences \\ Veeresh Nagar, Gulbarga.585105, India \\ ${ }^{2}$ Department of Microbiology, ESIC-MC-PGIMSR, Rajajinagar, \\ Bengaluru-560010, India \\ ${ }^{3}$ Department of Microbiology, ESIC-MC-PGIMSR, Rajajinagar, \\ Bengaluru-560010, India \\ *Corresponding author
}

\begin{abstract}
A B S T R A C T
Keywords

Candida albicans,

Non-C. albicans

Candida (NCAC),

Antifungal

Susceptibility

Profile.

\section{Article Info}

Accepted:

07 June 2016

Available Online:

10 July 2016

Candida spp. can cause a wide variety of infections in immunosuppressed patients of long term broad - spectrum antibiotics, steroids or other immunosuppressive agents, diabetes mellitus, AIDS, Malignancy, Neutropenia. Although the majority of infections are caused by Candida albicans, Non-C. albicans Candida (NCAC) species are emerging as important pathogen in humans. Our study included 130 clinical isolates which showed gram positive budding yeasts cells with or without pseudohyphae. $\mathrm{KOH}$ mount, germ tube, Carbohydrate assimilation test and fermentation test and antifungal susceptibility by disc diffusion method was done. Most of the Candida isolates were from sputum (45\%)samples followed by urine (24\%), endotracheal tip(14\%), vaginal swabs(9\%),blood (4\%)and pus(4\%).Female (57.6\%) more prone to Candida infections than male(42.3\%).54\% of the patients from whom candida was isolated had been treated with $>2$ antibiotics, $15 \%$ treated with $>3$ antibiotics, 17\% Diabetis Mellitus, $16 \%$ Neutropenia, $8 \%$ Tuberculosis and Treatment with Steroids,6\% Malignancy,2\% HIV. Most common Candida species isolated belonged to Candida albicans (29.2\%).C parapsilosis (15.3\%),C tropicalis (12.3\%), C glabrata (11.5\%),C krusei (9.2\%), C lusitanae (9.2\%), C guillermondi (8.46\%), $C$ dubliniensis (4.6\%).65\% of the total isolates were sensitive to Fluconazole and $82 \%$ of the total isolates were sensitive to Voriconazole.
\end{abstract}

\section{Introduction}

Candida is a part of the normal flora in healthy individuals, and is confined to the skin and mucosal surfaces of the oral cavity, gastrointestinal and urogenital tracts, and vagina. However, Candida spp. can cause a wide variety of infections in immuno- suppressed patients Candida species are responsible for around $80 \%$ of fungal infections in the hospital environment (Sullivan, 1996; Vinaya and Sharma, 2013; Sumitra and Maheshwari, 2014). Majority of infections are caused by Candida albicans, non- $C$. albicans Candida (NCAC) species 
like $C$ glabrata, $C$ tropicalis, $C$ parapsilosis, $C$ krusei. $C$ guillermondii, $C$ kefyr, or lusitaniae have been reported as cause of candidiasis (Sehal and Daniel, 2006; Chander, 2008; Kavitha, et al., 2014).

Candida causes a variety of infections like Oropharnygeal candidiasis, vaginal candidiasis, urinary tract infections, singleorgan infections including endophthalmitis, endocarditis, meningitis, pyelonephritis, septic arthritis; and disseminated infections with liver, kidney, spleen, and lung involvement (Chakrabarti and Shivprakash, 2005; Pfaller and Diekema, 2007; Manjunath, 2012).

Candida has emerged as the fourth most common cause of blood-borne infection. The mortality due to candida infections ranges from $14.5 \%$ to $49 \%$, and $C$ albicans is estimated to be responsible for $50-60 \%$ of the cases invasive candidiasis.

\section{Materials and Methods}

A total of 130 Candida species isolated from clinical samples like urine, pus, sputum, blood, oral swabs, vaginal swabs, endotracheal aspirates, endotracheal tips, were included in this study. The study was conducted from November 2012 to April 2014 in the department of Microbiology, ESIC MC PGI MSR Hospital Bangalore. Data of patients regarding the use of long term broad - spectrum antibiotics, steroids or other immunosuppressive agents, diabetes mellitus, AIDS, Malignancy, Neutropenia were collected at enrolment.

Examination of specimen was done by Gram staining, $10 \%$ and $40 \% \mathrm{KOH}$ mount, Cultural characteristics were noted on SDA with chloramphenicol, Germ tube test was done to detect elongated daughter cells without constriction, chlamydospore formation was noted on cornmeal agar with $1 \%$ tween 80 (by Dalmau technique) (Fig 1), further speciation was done by Carbohydrate assimilation test by Auxanographic plate method (Haley and standard modification) using Yeast nitrogen base and $20 \%$ sugars (Fig 2). Sugars used were glucose, sucrose, lactose, maltose, trehalose, cellobiose, raffinose, mellibiose, inositol, dulsitol, galactose, xylose. $3 \mathrm{ml}$ of Yeast suspension to $12 \mathrm{ml}$ of molten yeast nitrogen base agar was added and mixed by gently rotating the petriplate on the surface. With a sterile standard loop dipped in sugar is placed over the sterile disk and placed over the yeast suspension and agar medium and incubated at $37^{\circ} \mathrm{C}$ for $18-24$ hours and then examined for growth around each disk. A positive reaction was indicated by the presence of growth in the medium

Sugar fermentation test with $6 \%$ sugars was done for speciation.

Antifungal susceptibility test was done by Kirby- Bauer's disc diffusion method on glucose methylene blue Mueller Hinton agar (GM-MH) using commercially available antifungalcdiscs [Hi Media, Mumbai, India] fluconazole $(25 \mu \mathrm{g})$, Voriconazole $1 \mu \mathrm{g}$.(Fig $3)$. Zone diameters were interpreted as per the approved NCCLS (M44-A) guidelines. The quality control test was performed by using $C$. parapsilosis (ATCC 22019), and $C$. albicans (ATCC 90028).

\section{Zone Diameter Interpretive Standards}

\begin{tabular}{|l|l|lc|}
\hline Antifungal & Disk & \multicolumn{2}{|l|}{ Zone Diameter, Nearest } \\
agent & content & whole & S-DD \\
& & $\mathrm{R}$ & \\
& & $\leq 14$ & $15-18$ \\
\hline Fluconazole & $25 \mu \mathrm{g}$ & $\geq 19$ & $14-16$ \\
& & $\leq 13$ & \\
\hline Voriconazole & $1 \mu \mathrm{g}$ & $\geq 17$ & \\
& & & \\
\hline
\end{tabular}




\section{Results and Discussion}

Out of 130 Candida species isolated, Candida albicans (29.2\%) was the most common species isolated from the various clinical specimens. The Non Candida albicans species isolated were $C$ parapsilosis (15.3\%), C tropicalis (12.3\%), C glabrata( $11.5 \%), C$ krusei (9.2\%), C lusitanae (9.2\%), C guillermondi (8.46\%),C dubliniensis(4.6\%).

Candida species were isolated more commonly from sputum (45\%) followed by urine specimen (24\%), endotracheal tip $(14 \%)$, vaginal swabs $(9 \%)$, blood (4\%), and pus samples (4\%). Identification of risk factors is important way for prevention of diseases. In the present study, $54 \%$ of the patients from whom candida was isolated had been treated with $>2$ antibiotics, $15 \%$ had been treated with $>3$ antibiotics, $17 \%$ had Diabetis Mellitus, $16 \%$ had Neutropenia, $8 \%$ had Tuberculosis and $8 \%$ in those Treated with Steroids, $6 \%$ had Malignancy,2\% had HIV. In a study conducted by Kao AS et al., risk factors associated were antibacterial therapy (68\%), steroid therapy $(40 \%)$, neutropenia $(13 \%)$ while a study conducted by A.K. Verma showed association with broad-spectrum antibiotics (42.8\%) immunosuppressive therapy (23.8\%), neutropenia (14.3\%) which was similar to our present study. isolation of C albicans was $16 \%$ among HIV patients. $C$ tropicalis $(32 \%)$ and $C$ glabrata (32\%)most commonly isolated Candida spp in HIV infected individuals.

Fig.1 Demonstration of Single terminal chlamydospore of Candida in corn meal agar

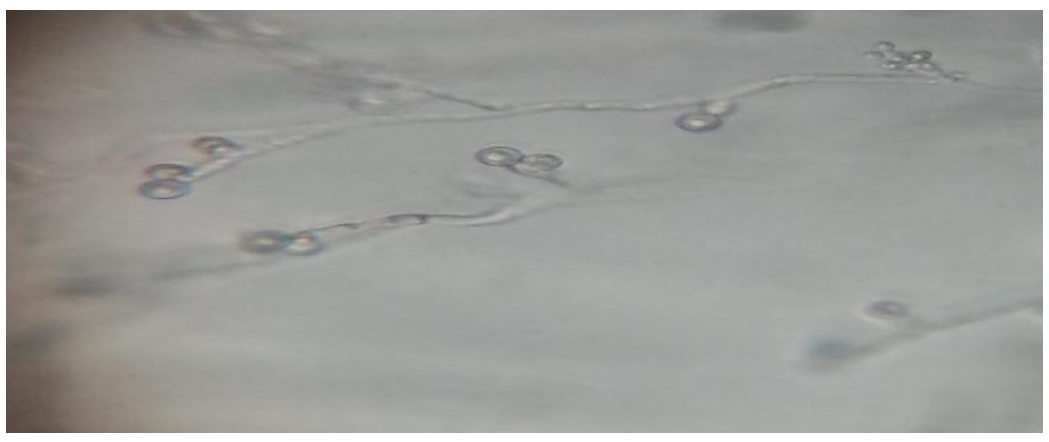

Fig.2 Carbohydrate assimilation test

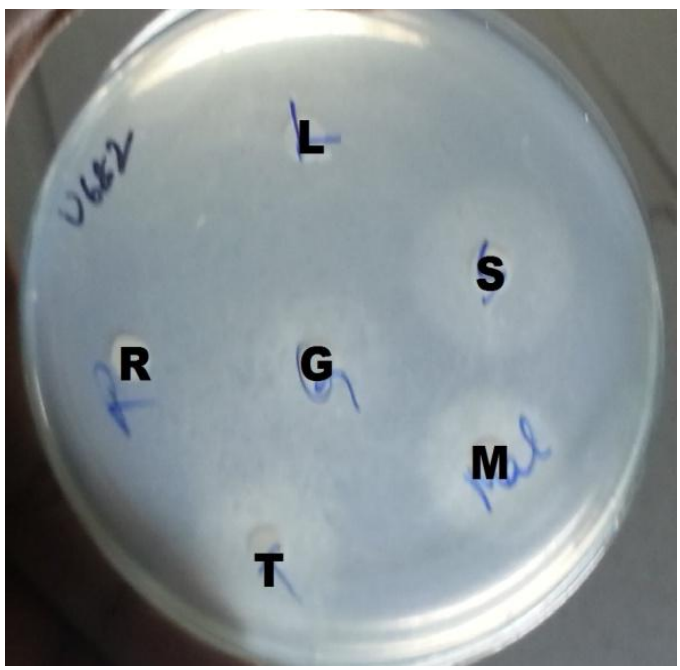


Fig.3 Antifungal Susceptibility test

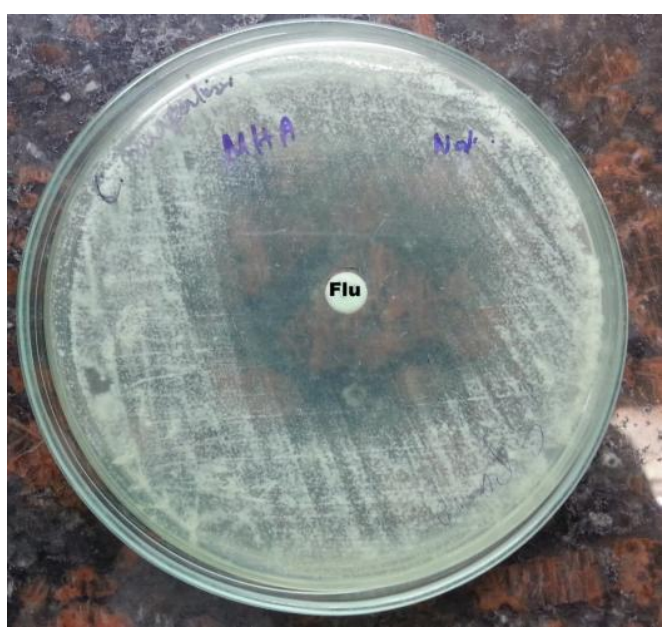

Fig.4 Gender Wise Distribution of the samples

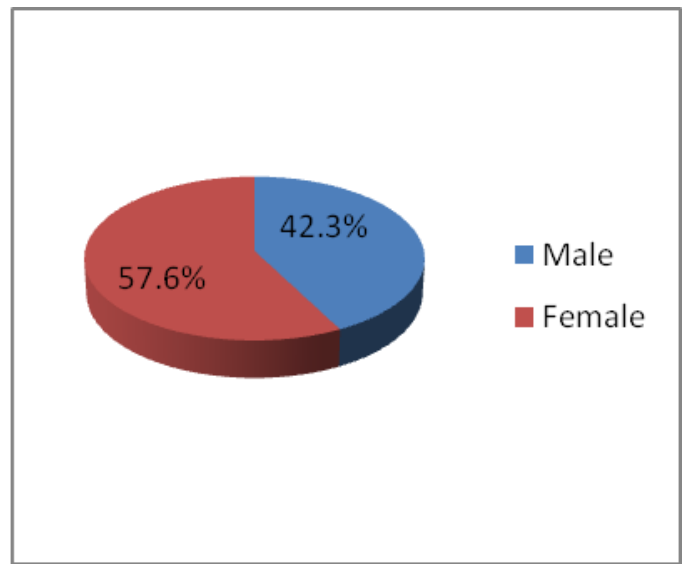

Fig.5 Age wise distribution of patients

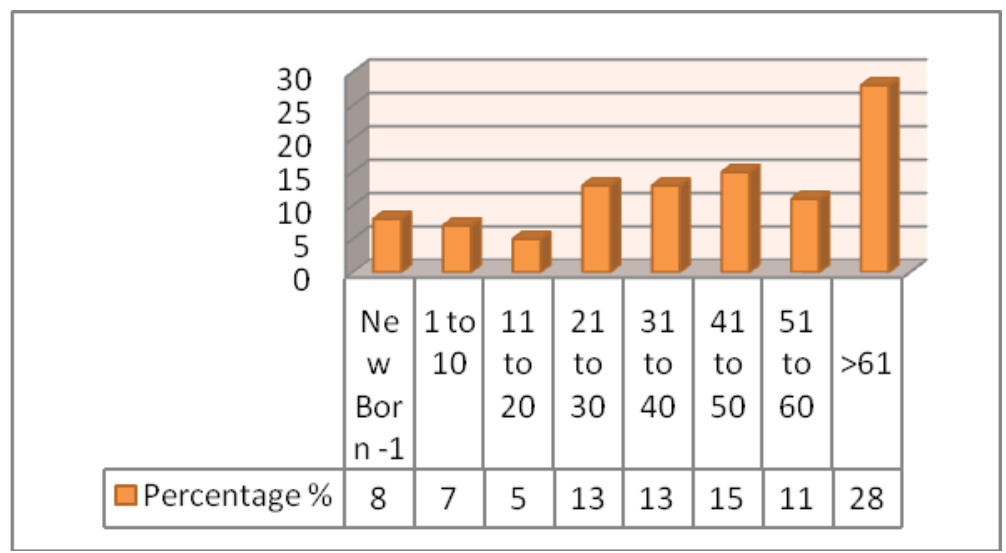


Fig.6 Ward Wise Distribution of samples

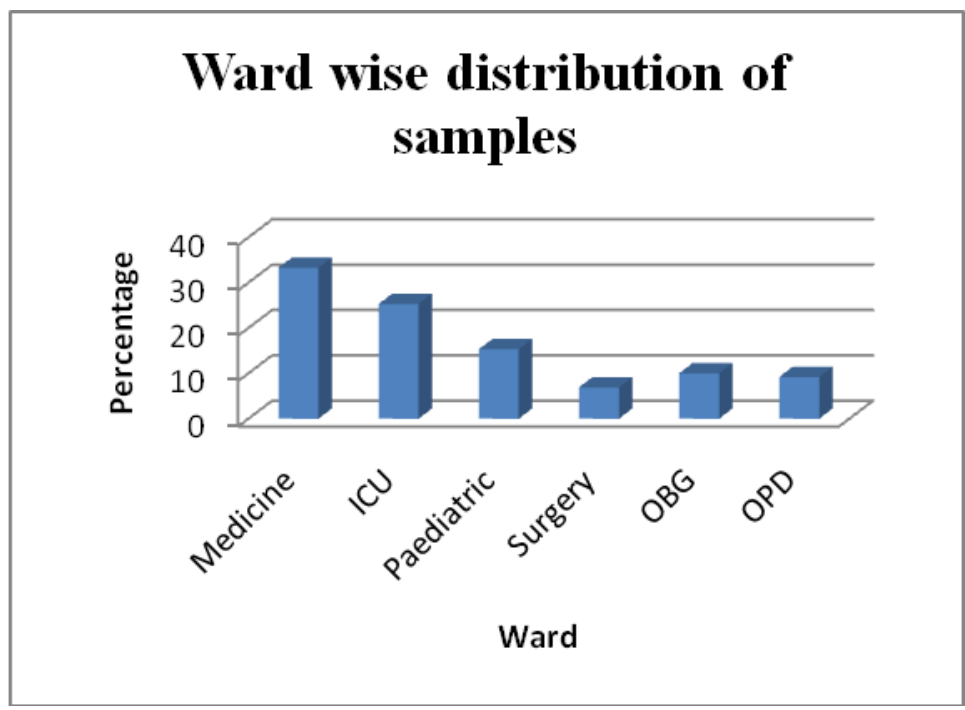

Fig.7 Sample wise distribution of Candida isolates

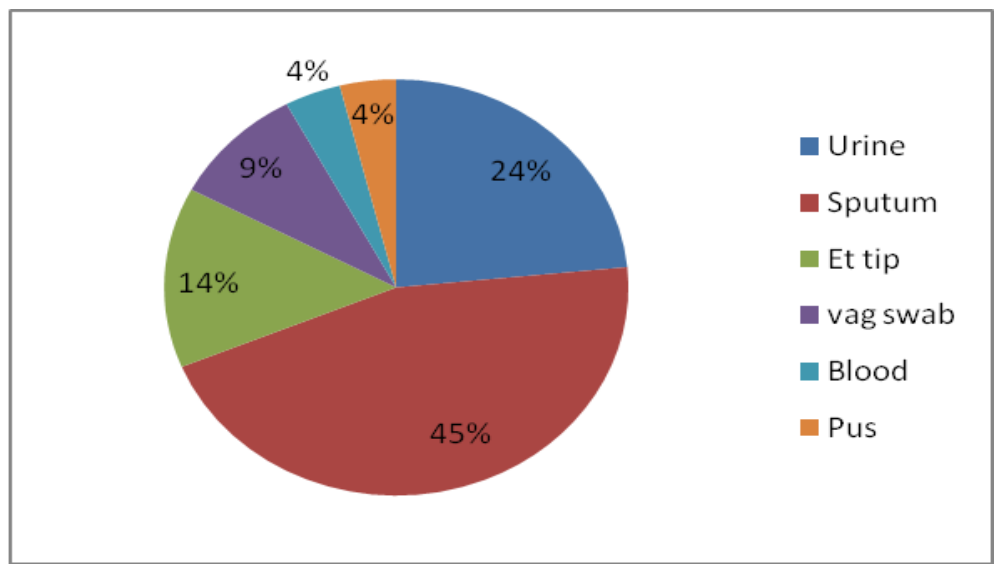

Fig.8 Distribution of different species of Candida

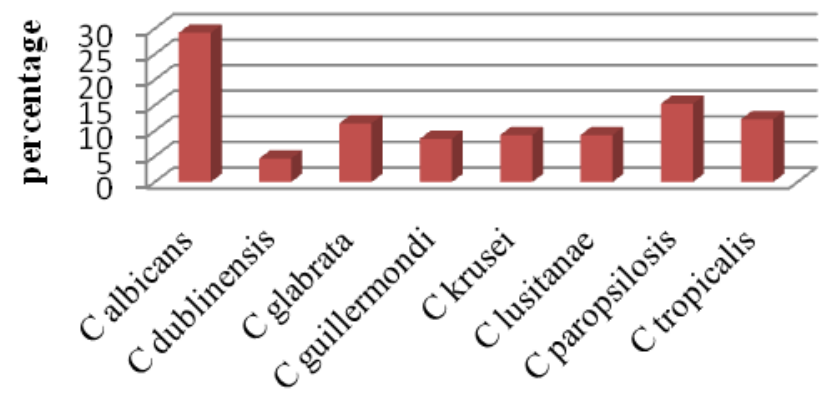

Candida species 
Fig.9 Risk Factors associated with Candida infections

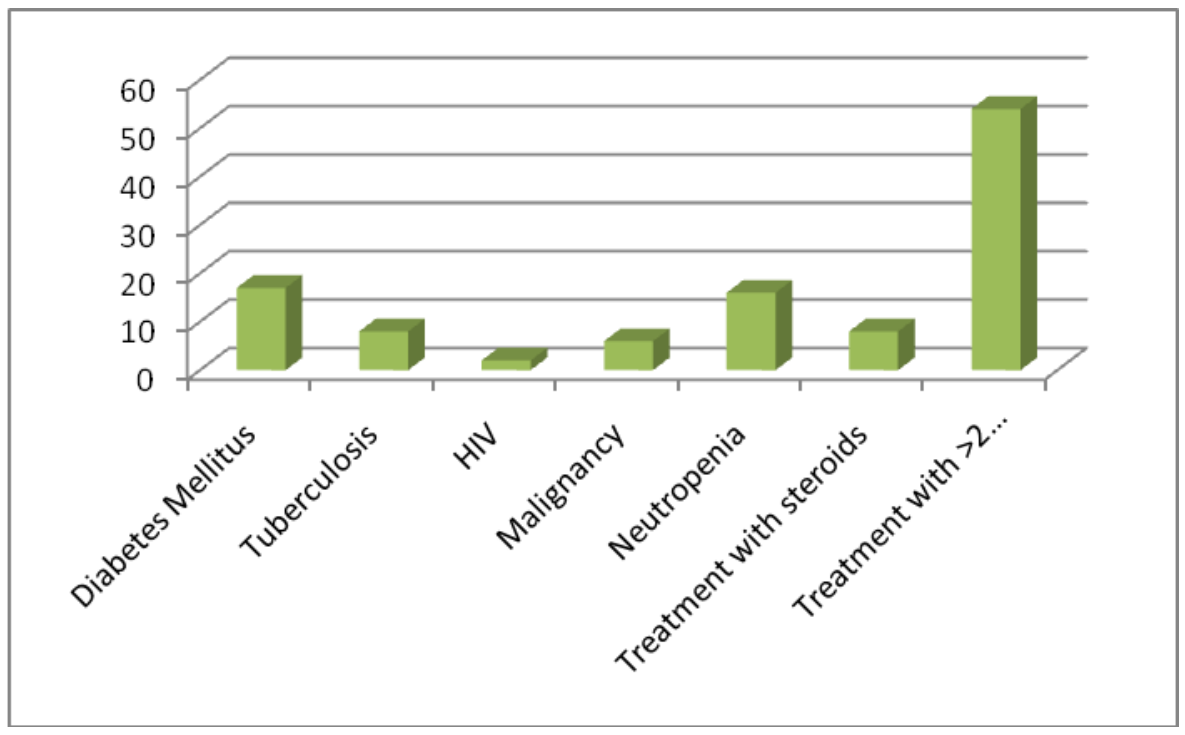

Age wise distribution of candida showed that $28 \%$ were $>60$ years, followed by $41-$ 50 years $(15 \%), \quad 21-30(13 \%), 31-40(13 \%)$ and 51-60 (11\%), NB-1 yr(8\%), 1-10(7\%) which was similar to a study conducted by Larone, (2002) who showed >60 (52.4\%), $51-60 \quad(23.8 \%), \quad 31-40 \quad(14.3 \%), \quad 41-50$ (4.8\%), 21-30 (4.8\%).

The study showed that female (57.6\%)were more prone to Candida infections than male (42.3\%). Antifungal susceptibility testing showed $65 \%$ of the isolates sensitive to Fluconazole and $35 \%$ resistant. $82 \%$ were sensitive and $18 \%$ resistant to Voriconazole.

$100 \%$ of $C$ dubliniensis isolates were sensitive to fluconazole and Voriconazole in the present study. $6(4.6 \%)$ of the total isolates were resistant to both fluconazole and Voriconazole.4 out of 6 isloates belonged to $C$ glabrata and 2 belonged to $C$ tropicalis.

$6(4.6 \%)$ isolates of the total 130 isolates were resistant to fluconazole but sensitive to Voriconazole. Of which 1 isolate was $C$. albicans, $\quad C$. guillermondi(1each), $C$. glabrata and $C$. krusei(2 each).
In conclusion, the present study showed that $C$ albicans was the predominant species isolated. Among the non Candida albicans $C$ parapsilosis was predominantly isolated followed by $C$ tropicalis, $C$ glabrata, $C$ krusei,C lusitanae, $C$ guillermondi, $C$ dubliniensis.

Voriconazole seemed to be superior to fluconazole with a better susceptibility in the fluconazole resistant strains also. The triazoles remain active against many of isolates and voriconazole remains sensitive to the fluconazole resistant strains, also Fluconazole can be continued as the first line antifungal agent for treating suspected cases of uncomplicated candidiasis.

Voriconazole can be reserved for refractory cases of candidiasis. But whenever there is lapses in infection control precautions coupled with broad use of fluconazole, more fluconazole-resistant strain of endemic species may emerge

\section{Acknowledgement}

I thank all the staff of department of Microbiology, ESIC MC and PGIMSR, 
Bengaluru for their help and support while conducting the study.

\section{References}

Chander, J. 2008. Candidiasis in Text book of medical mycology. $3^{\text {rd }}$ edition.: Mehta pub, 267-290.

Chakrabarti, A., Shivprakash, M. 2005. Laboratory Diagnosis. In Medical Mycology Laboratory Procedures, 1770.

Kavitha, H., Anuradha, K., Venkatesha, D. 2014. Comparison of Susceptibility of Various Candida Species Isolated From Neonatal Septicaemia to Voriconazole and Fluconazole. J. Pharm. Biol. Sci., 9(2):78-81.

Larone, D.H. 2002. Medically Important Fungi; A guide to identification, Washington DC. ASM Press, 328-329.

Sehal, E., Daniel, E. 2006. Candidiasis in Topley and Wilson's Microbiology and Microbial Infections, Medical Mycology. $10^{\text {th }}$ edition, ASM Press, 580-613.

Manjunath, V., Vidya, G.S., Sharma, S., Prakash, M.R., Murugesh. 2012. Speciation of Candida by Hicrome agar and Sugar assimilation test in both HIV infected and non infected patients. Int. J. Biol. Med. Res., 3(2): 1778-1782.

Pfaller, M.A., Diekema, D.J. 2007. Epidemiology of Invasive Candidiasis: A Persistent Public Health Problem. Clin. Microbiol. Rev., 20(1): 133.

Sumitra, D., Maheshwari, M. 2014. Speciation of Candida Species Isolated From Clinical Specimens by Using Chrom Agar and Conventional Methods. Int. J. Sci. Res. Pub., 4(3): 2.

Shettar, S.K., Asha, B.P., Shobha, D.N., Shepur, T.A., Mythri, B.A., Santosh G. Evaluation of HiCrome differential agar for speciation of candida. J. Acad. Med. Sci., 2(3): 101-104.

Sullivan, D.J., Henmans, M.C., Moran, G.I., Lorraine, C., O'neill, Bennett, D.E, et al. 1996. Molecular genetic approaches to identification, epidemiology and taxonomy of non-albicans Candida species. J Med. Microbiol., 44: 399-408.

Vinaya, B., Sharma, S.M., Veena, S., Shastry, C.S., Vaman, R., Shilpa, M.S. 2013. Prevalence Of Candida Associated Denture Stomatitis(Cads) and Speciation Of Candida Among Complete Denture Wearers Of South West Coastal Region Of Karnataka. Nitte University J. Hea Sci., 3(3): 59-63.

\section{How to cite this article:}

Natasha, M.V., Y. Raksha and Navaneeth, B.V. 2016. Speciation of Candida Isolated from Various Clinical Samples and their Antifungal Susceptibility Profile in a Tertiary Care Hospital. Int.J.Curr.Microbiol.App.Sci. 5(7): 54-60. doi: $\underline{\text { http://dx.doi.org/10.20546/ijcmas.2016.507.004 }}$ 